\title{
Gas-phase laboratory formation of large, astronomically relevant PAH-organic molecule clusters
}

\author{
Xiaoyi $\mathrm{Hu}^{1,2,3}$, Yuanyuan Yang ${ }^{1,2,3}$, Congcong Zhang ${ }^{4}$, Yang Chen $^{3}$, Junfeng Zhen ${ }^{1,2}$, and Liping Qin ${ }^{1,2}$ \\ ${ }^{1}$ CAS Key Laboratory of Crust-Mantle Materials and Environment, University of Science and Technology of China, Hefei 230026, \\ PR China \\ e-mail: jfzhen@ustc.edu.cn \\ 2 CAS Center for Excellence in Comparative Planetology, University of Science and Technology of China, Hefei 230026, \\ PR China \\ ${ }^{3}$ CAS Center for Excellence in Quantum Information and Quantum Physics, Hefei National Laboratory for Physical Sciences at the \\ Microscale, and Department of Chemical Physics, University of Science and Technology of China, Hefei 230026, PR China \\ ${ }^{4}$ CAS Key Laboratory for Research in Galaxies and Cosmology, Department of Astronomy, University of Science and Technology \\ of China, Hefei 230026, PR China
}

Received 28 May 2021 / Accepted 23 September 2021

\begin{abstract}
Polycyclic aromatic hydrocarbon (PAH) molecules may play an essential role in the prebiotic compound evolution network in interstellar clouds. In this work, an experimental study of large, astronomically relevant PAH-organic molecule clusters is presented. With the initial molecular precursors dicoronylene (DC; $\mathrm{C}_{48} \mathrm{H}_{20}$ )-pyroglutamic acid $\left(\mathrm{Pga}, \mathrm{C}_{5} \mathrm{H}_{7} \mathrm{NO}_{3}\right)$, DC-proline $\left(\operatorname{Pro} ; \mathrm{C}_{5} \mathrm{H}_{9} \mathrm{NO}_{2}\right)$, and DC-pyroglutaminol (Pgn; $\mathrm{C}_{5} \mathrm{H}_{9} \mathrm{NO}_{2}$ ), our experiments indicate that $\mathrm{PAH}$-organic molecule cluster cations (e.g., $(\mathrm{Pga})_{(1-2)} \mathrm{C}_{48} \mathrm{H}_{n}^{+}$, $(\text { Pro })_{(1-2)} \mathrm{C}_{48} \mathrm{H}_{n}^{+}$, and $(\mathrm{Pgn})_{(1-6)} \mathrm{C}_{48} \mathrm{H}_{n}^{+}$) and carbon cluster-organic molecule cluster cations (e.g., (Pga) $\mathrm{C}_{48}^{+}$, $(\text {Pro })_{(1-2)} \mathrm{C}_{48}^{+}$, and $\left.(\mathrm{Pgn})_{(1-6)} \mathrm{C}_{48}^{+}\right)$are gradually formed through an ion-molecule collision reaction pathway in the presence of a strong galactic interstellar radiation field. These laboratory studies provide a gas-phase growth route toward the formation of complex prebiotic compounds in a bottom-up growth process, as well as insight into their chemical-evolution behavior in the interstellar medium.
\end{abstract}

Key words. astrochemistry - molecular processes - methods: laboratory: molecular - ultraviolet: ISM - ISM: molecules

\section{Introduction}

In the interstellar medium (ISM), the infrared (IR) broadband (on the order of $20-40 \mathrm{~cm}^{-1}$ ) features at 3.3, 6.2, 7.7, 8.6, and $11.2 \mu \mathrm{m}$ are generally attributed to the family of polycyclic aromatic hydrocarbon (PAH) molecules (e.g., Sellgren 1984; Puget \& Leger 1989; Allamandola et al. 1989). In particular, two nitrile-group-functionalized PAHs, 1-cyanonaphthalene and 2cyanonaphthalene, have recently been detected in the ISM. Both bicyclic ring molecules were observed in the TMC-1 molecular cloud (McGuire et al. 2021). The presence of PAH clusters and very small dust grains are also determined by the interstellar IR features (Van Diedenhoven et al 2004; Rapacioli et al. 2005; Berné et al. 2007; Pilleri et al. 2015). In the molecular cloud, PAH clusters are believed to be the self-assembled intermediaries between PAHs and other coexisting molecules, and PAH radicals have been discussed as being the driving force behind such transitions (Rapacioli et al. 2006; Rhee et al. 2007; Zhen 2019; Gavilan et al. 2020).

Interstellar complex organic molecules are thought to be the building blocks of more complex prebiotic compounds (Dulieu et al. 2019). Organic molecules are known to exist in star-forming regions and in protoplanetary disks where planets are formed (Herbst \& van Dishoeck 2009). Hollis et al. (2000) reported the first detection of interstellar glycolaldehyde $\left(\mathrm{CH}_{2} \mathrm{OHCHO}\right)$ in the gas phase of the star-forming cloud Sagittarius B2(N) and, more recently, toward a star-forming hot molecular core, G31.41+0.31 (Beltrán et al. 2009); both sources are rich in organic molecules. Observations have established the existence of ethyl cyanide $\left(\mathrm{CH}_{3} \mathrm{CH}_{2} \mathrm{CN}\right)$, acetone $\left(\mathrm{CH}_{3} \mathrm{COCH}_{3}\right)$, and possibly the amino acid glycine $\left(\mathrm{NH}_{2} \mathrm{CH}_{2} \mathrm{COOH}\right)$ in the interstellar clouds (Snyder 1997). Favre et al. (2018) also reported the first detection of the simplest organic acid (formic acid, $\mathrm{HCOOH}$ ) toward the TW Hydrae protoplanetary disk. However, free organic molecules in the gas phase are more fragile with UV irradiation and cannot survive in a harsh environment (e.g., a galactic interstellar radiation field) of the ISM. As in the HI regions $(5.0<h v<13.6 \mathrm{eV})$, the energy of a single photon is capable of dissociating these molecules (Herbst \& van Dishoeck 2009; Woods et al. 2012).

Polycyclic aromatic hydrocarbon molecules and their derivatives may play an important role in the evolution network of prebiotic compounds (e.g., Puget \& Leger 1989; Allamandola 2011; Tielens 2013). As hosts, large PAH species, PAH clusters, or very small dust grains can protect these gas-phase organic molecules avoid UV dissociation and survive in areas of high luminosity in interstellar environments (Tielens 2013; Zhen 2019; Gavilan et al. 2020). However, the chemical process of the gas-phase free organic molecules accreting onto large PAH species, PAH clusters, or very small dust grains is unclear.

In this paper, the gas-phase formation of large, astronomically relevant PAH-organic molecule clusters is studied. The main objective is to investigate the stability and formation mechanisms of PAH-organic molecule clusters in the collision reaction process (between cationic $\mathrm{PAH}$ species and organic molecules). We explore large $\mathrm{PAH}$ cations $\left(\mathrm{DC}^{+}, \mathrm{C}_{48} \mathrm{H}_{20}^{+}\right.$) with an organic molecule as the initial precursor. Experiments are performed using a quadrupole mass filter (QMF) or quadrupole 


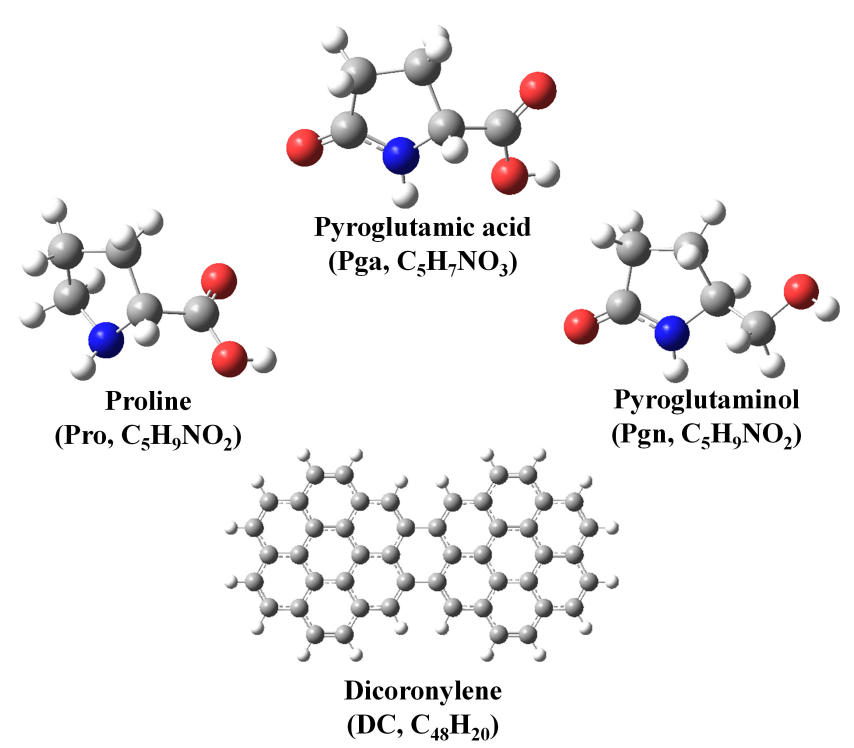

Fig. 1. Molecular geometry and formula of pyroglutamic acid (Pga, $\mathrm{C}_{5} \mathrm{H}_{7} \mathrm{NO}_{3}, 129 \mathrm{amu}$ ), proline (Pro, $\mathrm{C}_{5} \mathrm{H}_{9} \mathrm{NO}_{2}, 115 \mathrm{amu}$ ), pyroglutaminol $\left(\mathrm{Pgn}, \mathrm{C}_{5} \mathrm{H}_{9} \mathrm{NO}_{2}, 115 \mathrm{amu}\right.$ ), and dicoronylene (DC, $\left.\mathrm{C}_{48} \mathrm{H}_{20}, 596 \mathrm{amu}\right)$.

ion trap (QIT) source setup, analyzed by time-of-flight (TOF) mass spectrometry, together with two heatable ovens for the large gas-phase PAHs and the organic molecules, respectively.

As shown in Fig. 1, pyroglutamic acid $\left(\mathrm{Pga}, \mathrm{C}_{5} \mathrm{H}_{7} \mathrm{NO}_{3}\right)$, proline (Pro, $\left.\mathrm{C}_{5} \mathrm{H}_{9} \mathrm{NO}_{2}\right)$, and pyroglutaminol $\left(\mathrm{Pgn}, \mathrm{C}_{5} \mathrm{H}_{9} \mathrm{NO}_{2}\right)$ are used as the reactants and as examples of interstellar complex organic molecules based on the following three considerations (Herbst \& van Dishoeck 2009). First, pyroglutamic acid, proline, and pyroglutaminol are a family of typical organic molecules. Pyroglutamic acid is the derivative product of glutamic (when one $\mathrm{H}_{2} \mathrm{O}$ is lost) and has a cyclic structure. Proline and pyroglutaminol contain a cyclic structure and are isomers; both are derivative products of pyroglutamic acid (when one oxygen atom is replaced in different locations). Second, pyroglutamic acid (16 atoms, $129 \mathrm{amu}$ ), proline (17 atoms, $115 \mathrm{amu}$ ), and pyroglutaminol (17 atoms, $115 \mathrm{amu}$ ) have relatively large sizes for organic molecules. Finally, pyroglutamic acid, proline, and pyroglutaminol are suitable for heating in the oven, which evaporates them into the gas phase efficiently. Dicoronylene is an all-benzenoid $\mathrm{PAH}$ with a size $(48 \mathrm{C}$ atoms and $20 \mathrm{H}$ atoms; $596 \mathrm{amu}$ ) in the astrophysically relevant range (Croiset et al. 2016; Zhen et al. 2018).

\section{Experimental methods}

The following is a brief description of the experiment (Zhen 2019). DC was evaporated by heating (using Kentax, with purity better than $99.5 \%$, at $\sim 580 \mathrm{~K}$ ) in the ion source chamber, ionized, and then transported into the ion trap. A second oven was located under the ion trap to vaporize the organic molecules (pyroglutamic acid, proline, and pyroglutaminol powder, with purity better than $99 \%$, at $\sim 380,360$, and $310 \mathrm{~K}$ ) in the ion trap chamber, which could effuse continuously toward the center of the ion trap. The third harmonic of an Nd:YAG laser, $355 \mathrm{~nm}, \sim 6 \mathrm{~ns}$, operated at $10 \mathrm{~Hz}$, was used, and a beam shutter determined the interaction time of the light with the trapped ions. A high-precision delay generator controlled the full-timing sequence.

Our setup operated with a typical frequency of $0.1 \mathrm{~Hz}$. The ion gate opened for $0.0-5.8 \mathrm{~s}$, allowing the ion trap to be filled with a certain amount of ions. The beam shutter opened for 5.8-6.8 s (i.e., typically $\sim 10$ pulses), allowing the laser irradiation of the trapped ions. With a time delay period of $6.8-9.88 \mathrm{~s}$ and collision reaction times amounting to $3.0 \mathrm{~s}$, the trapped new photo-formed ions collided with neutral organic molecules to form new cluster cations. Then, a negative square pulse $(9.88 \mathrm{~s})$ was applied to the end cap of the ion trap, accelerating the ions out of the trap and into the TOF region, where the resulting mass fragments were measured. The resulting mass spectra are shown in Figs. 2-4.

\section{Experimental results}

Figure 2 shows the resulting mass spectrum of trapped DCpyroglutamic acid cluster cations upon interactions with $355 \mathrm{~nm}$ photons at $3.0 \mathrm{~mJ}(5.8-6.8 \mathrm{~s})$ as well as upon collision reactions with neutral pyroglutamic acid molecules (6.8-9.88 s). A series of cationic clusters were generated, and many new mass peaks are observed.

More details regarding the newly formed species are provided in Fig. 2. The first group of formed species are $\mathrm{C}_{n}^{+}, n=[44$, 48] (e.g., with a 24 amu difference), which are separated by a mass difference equivalent of two carbon atoms. The dehydrogenated DC (DDC) cations, $\mathrm{C}_{48} \mathrm{H}_{(1-19)}^{+}(\mathrm{m} / z=577-595)$, are produced from the photo-fragmentation process of the original $\mathrm{DC}^{+}$(Lifshitz 2000; Castellanos et al. 2018; Zhen 2019). We note that in this work, since we only have the dehydrogenation process, the obtained $\mathrm{C}_{48}$ carbon cluster species are mainly in graphene structures. However, with the $\mathrm{C}_{2}$ loss process, the structure of smaller carbon cluster molecules $\left(\mathrm{C}_{40}-\mathrm{C}_{46}\right.$ cations $)$ obtained by laser photolysis may still be in graphene structures or may tend to be in open-cage or semi-cage structures and may contain a number of fullerene-like carbon cage structures (Lifshitz 2000; Zhen et al. 2014; Castellanos et al. 2018). We only considered $\mathrm{DDC}^{+}\left(\mathrm{C}_{48} \mathrm{H}_{(1-19)}^{+}\right)$and pure carbon clusters $\left(\mathrm{C}_{n}^{+}, n=[40,48]\right)$ in the next step of reactions.

The second group of formed species are $(\mathrm{Pga}) \mathrm{C}_{n}^{+}, n=[44$, 48] and $(\mathrm{Pga}) \mathrm{DDC}^{+}$, formed through the collision reaction between fragmented $\mathrm{DC}$ cations $\left(\mathrm{C}_{n}^{+}\right.$and $\left.\mathrm{DDC}^{+}\right)$and pyroglutamic acid molecules. Similarly, the third group of formed species are $(\mathrm{Pga})_{2} \mathrm{DDC}^{+}$, formed through $(\mathrm{Pga}) \mathrm{DDC}^{+}$collision reactions with pyroglutamic acid molecules. The fourth group of formed species are $(\mathrm{Pga})_{3} \mathrm{DDC}^{+}$, formed through the collision reaction of $(\mathrm{Pga})_{2} \mathrm{DDC}^{+}$with pyroglutamic acid molecules.

From the mass spectrum presented in Fig. 2, the intensity ratio of $(\mathrm{Pga}) \mathrm{DDC}^{+} / \mathrm{DDC}^{+}$is smaller than $(\mathrm{Pga}) \mathrm{C}_{n}^{+} / \mathrm{C}_{n}^{+}$. This may suggest that the dehydrogenated $\mathrm{PAH}$ cations have a lower chemical reactivity than carbon cluster cations during collision reactions with pyroglutamic acid molecules.

We present the photo-fragmentation and the formation pathway for the DC-pyroglutamic acid system: in the time range of 5.8-6.8 s, the photo-fragmentation pathway upon laser irradiation for DC cations is as follows:

$$
\mathrm{C}_{48} \mathrm{H}_{20}^{+} \stackrel{h v}{\longrightarrow} \mathrm{C}_{48} \mathrm{H}_{(0-19)}^{+} ; \mathrm{C}_{48}+\stackrel{h v}{\longrightarrow} \mathrm{C}_{n}^{+}+\mathrm{C}_{2} .
$$

In the time range of $6.8-9.88 \mathrm{~s}$, the new cluster cations are formed through a collision formation reaction between fragmented cations (as shown above) and pyroglutamic acid molecules. The formation pathways are summarized as follows:

$$
\begin{aligned}
(\mathrm{Pga})_{m-1} \mathrm{DDC}^{+} & \stackrel{\text { Pga }}{\longrightarrow}(\mathrm{Pga})_{m} \mathrm{DDC}^{+}, m=[1,3] \\
\mathrm{C}_{n}^{+} & \stackrel{\text { Pga }}{\longrightarrow}(\mathrm{Pga}) \mathrm{C}_{n}^{+} .
\end{aligned}
$$




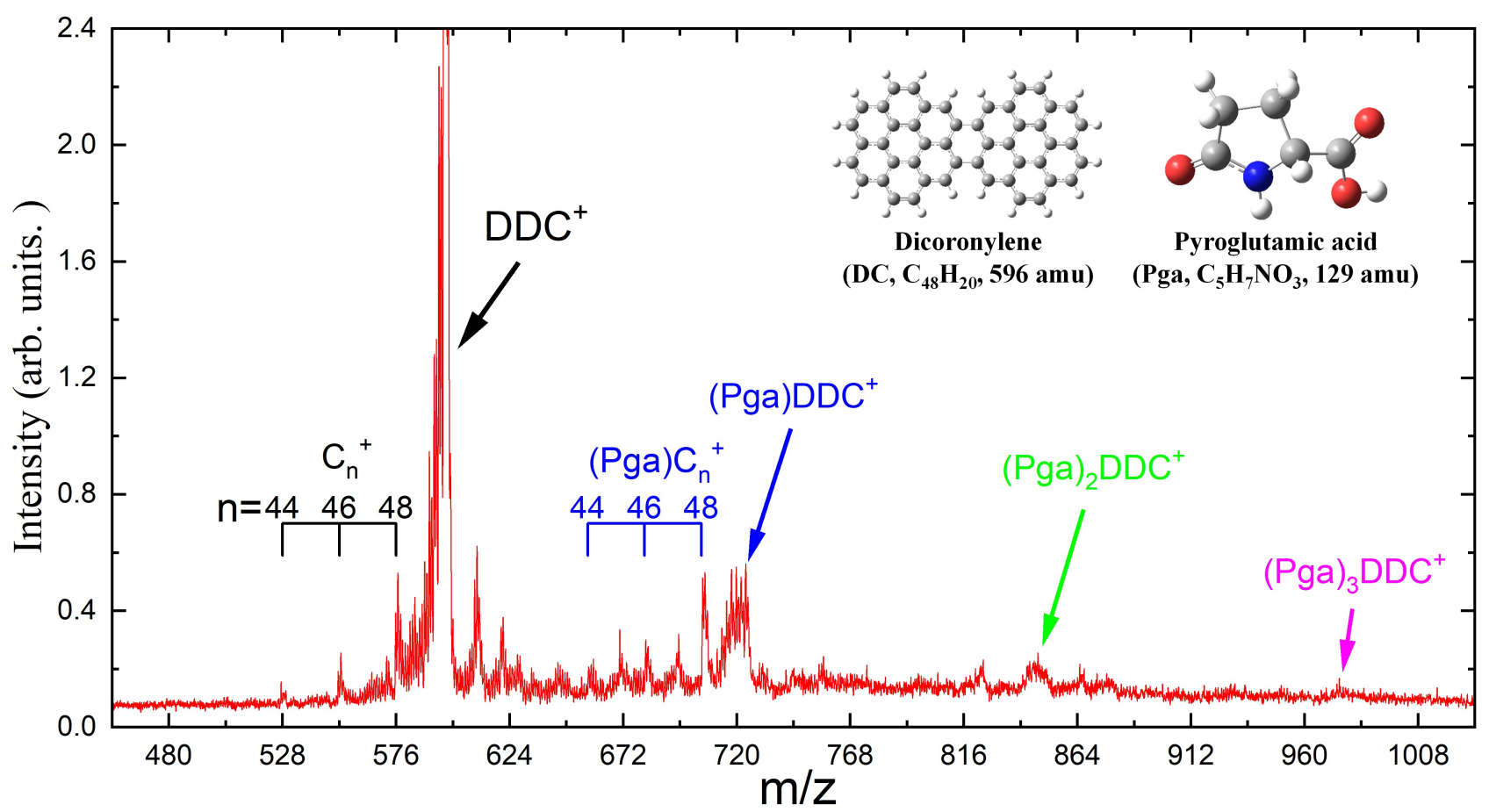

Fig. 2. Formation pattern of DC-pyroglutamic acid ( $\left.\mathrm{Pga}, \mathrm{C}_{5} \mathrm{H}_{7} \mathrm{NO}_{3}\right)$ cluster cations, trapped in QIT with laser irradiation $(355 \mathrm{~nm}, 3.0 \mathrm{~mJ}$, irradiation times amounting to $1.0 \mathrm{~s}$, from $5.8-6.8 \mathrm{~s}$ ) and then a collision reaction in the period $6.8-9.88 \mathrm{~s}$.

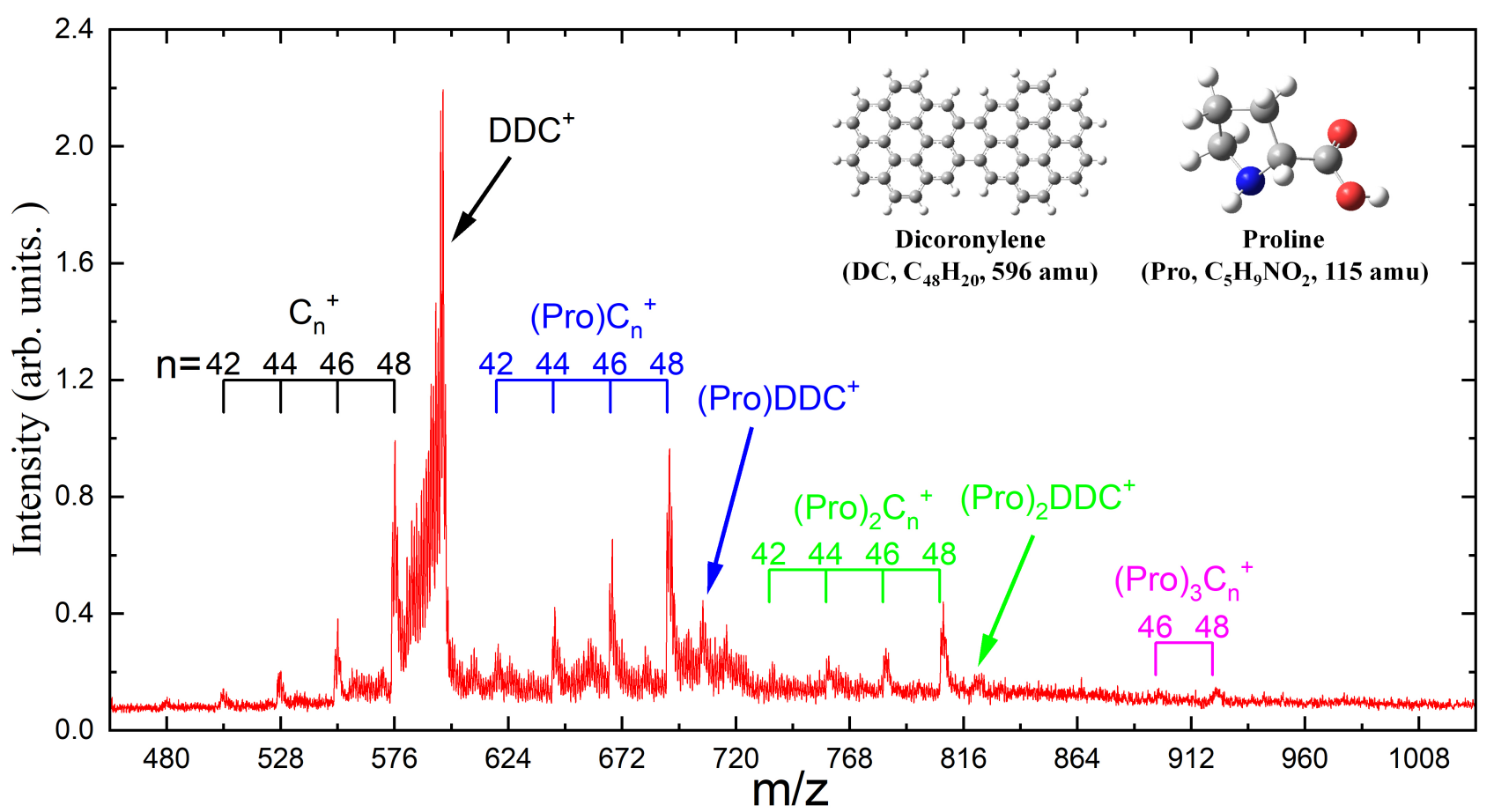

Fig. 3. Formation pattern of DC-proline (Pro, $\mathrm{C}_{5} \mathrm{H}_{9} \mathrm{NO}_{2}$ ) cluster cations, trapped in QIT with laser irradiation ( $355 \mathrm{~nm}, 4.0 \mathrm{~mJ}$, irradiation times amounting to $1.0 \mathrm{~s}$, from $5.8-6.8 \mathrm{~s}$ ) and then a collision reaction in the period $6.8-9.88 \mathrm{~s}$.

In Fig. 3, the resulting mass spectrum of trapped DC-proline cluster cations upon interaction with $355 \mathrm{~nm}$ photons at $4.0 \mathrm{~mJ}$ $(5.8-6.8 \mathrm{~s})$ and then upon a collision reaction with neutral proline molecules $(6.8-9.88 \mathrm{~s})$ is presented. A series of cationic clusters were generated, and many more new mass peaks are observed.

More detail on the new formation species is given in Fig. 3. The first group of formed species are $\mathrm{C}_{n}^{+}, n=[42,48]$ and
$\mathrm{DDC}^{+}, \mathrm{C}_{48} \mathrm{H}_{(1-19)}^{+}$, which were produced from the fragmentation process of the original $\mathrm{DC}^{+}$(Lifshitz 2000; Castellanos et al. 2018). The second group of formed species are (Pro) $\mathrm{C}_{n}^{+}$, $n=[42,48]$ and (Pro)DDC ${ }^{+}$, formed through the collision reaction between fragmented DC cations $\left(\mathrm{C}_{n}^{+}\right.$and $\left.\mathrm{DDC}^{+}\right)$and proline molecules. Similarly, the third group of newly formed species are (Pro) ${ }_{2} \mathrm{C}_{n}^{+}, n=[42,48]$ and (Pro) ${ }_{2} \mathrm{DDC}^{+}$, formed through (Pro) $\mathrm{C}_{n}^{+}$ and (Pro)DDC ${ }^{+}$collision reactions with proline molecules. The 


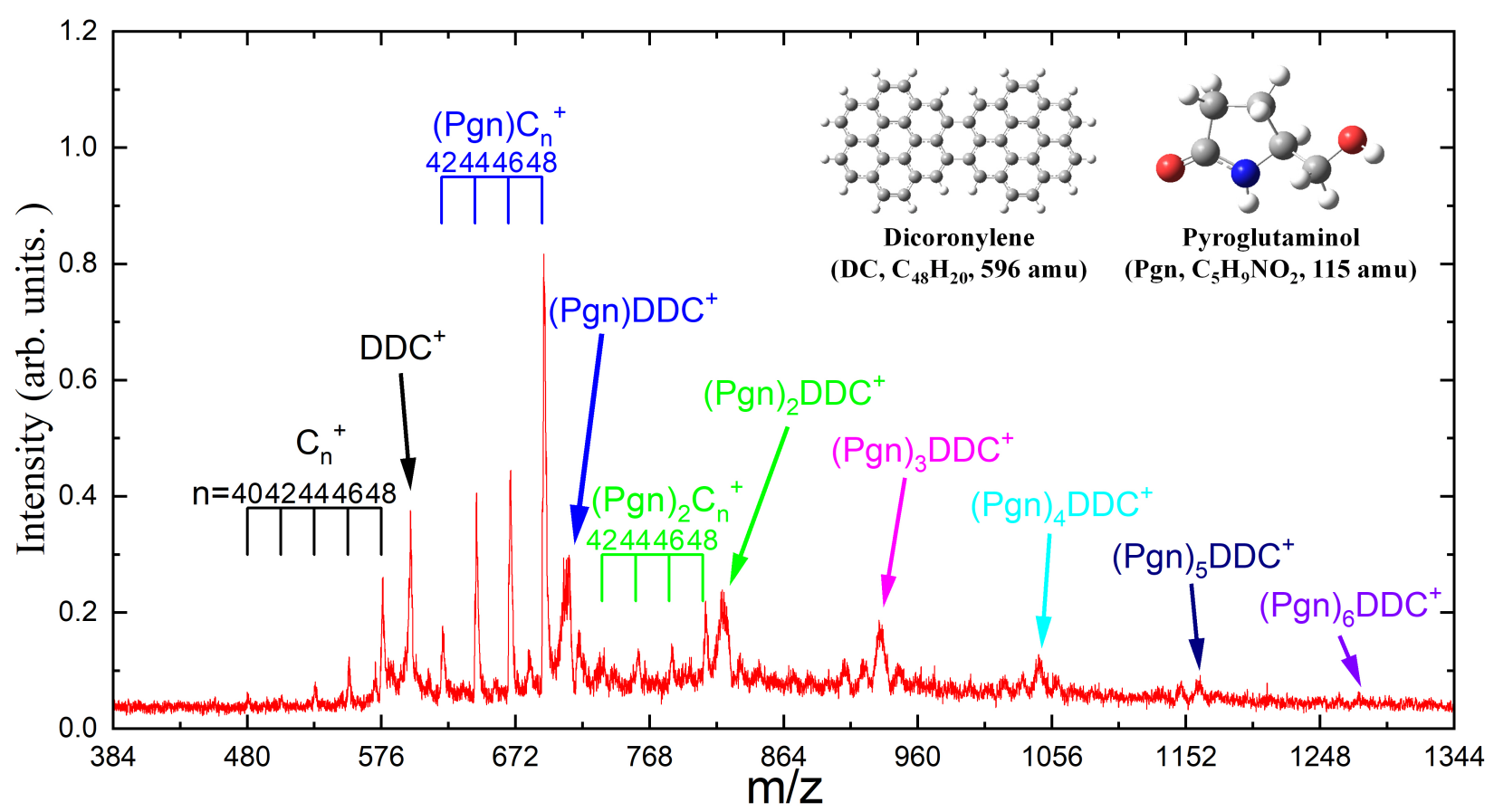

Fig. 4. Formation pattern of DC-pyroglutaminol (Pgn, $\left.\mathrm{C}_{5} \mathrm{H}_{9} \mathrm{NO}_{2}\right)$ cluster cations, trapped in QIT with laser irradiation $(355 \mathrm{~nm}, 5.0 \mathrm{~mJ}$, irradiation times amounting to $1.0 \mathrm{~s}$, from 5.8-6.8 s) and then a collision reaction in the period 6.8-9.88 $\mathrm{s}$.

fourth group of formed species are $(\mathrm{Pro})_{3} \mathrm{C}_{n}^{+}, n=[46,48]$, also formed through the collision reaction of (Pro $)_{2} \mathrm{DDC}^{+}$with proline molecules.

From the mass spectrum presented in Fig. 3, the intensity ratio of (Pro)DDC ${ }^{+} / \mathrm{DDC}^{+}$is smaller than (Pro) $\mathrm{C}_{n}^{+} / \mathrm{C}_{n}^{+}$. This may suggest that the dehydrogenated $\mathrm{PAH}$ cations have a lower chemical reactivity than carbon cluster cations during reactions with proline molecules.

We present the photo-fragmentation and formation pathway for the DC-proline system: In the time range of 5.8-6.8 s, the photo-dissociation channel upon laser irradiation for DC cations should be the same as that shown above; in the time range of 6.8 $9.88 \mathrm{~s}$, the new cluster cations are formed through a collision formation reaction between fragmented DC cations (as shown above) and proline molecules. The formation reaction pathways are summarized as follows:

$$
\begin{gathered}
(\text { Pro })_{m-1} \mathrm{DDC}^{+} \stackrel{\text { Pro }}{\longrightarrow}(\text { Pro })_{m} \mathrm{DDC}^{+}, m=[1,2] \\
(\text { Pro })_{m-1} \mathrm{C}_{n}^{+} \stackrel{\text { Pro }}{\longrightarrow}(\text { Pro })_{m} \mathrm{C}_{n}^{+}, m=[1,3] .
\end{gathered}
$$

In Fig. 4, the resulting mass spectrum of trapped DCpyroglutaminol cluster cations upon interaction with $355 \mathrm{~nm}$ photons at $5.0 \mathrm{~mJ}(5.8-6.8 \mathrm{~s})$ and then a collision reaction with neutral pyroglutaminol molecules $(6.8-9.88 \mathrm{~s})$ is presented. A series of cationic clusters were generated, and many more new mass peaks are observed.

More detail on the new formation species is given in Fig. 4. The first group of formed species are $\mathrm{C}_{n}^{+}, n=[42,48]$ and $\mathrm{DDC}^{+}, \mathrm{C}_{48} \mathrm{H}_{(1-19)}^{+}$, which were produced from the fragmentation process of the original $\mathrm{DC}^{+}$(Lifshitz 2000; Castellanos et al. 2018). The second group of formed species are (Pgn) $\mathrm{C}_{n}^{+}$, $n=[42,48]$ and (Pgn)DDC ${ }^{+}$, formed through the collision reaction of fragmented DC cations $\left(\mathrm{C}_{n}^{+}\right.$and $\left.\mathrm{DDC}^{+}\right)$with pyroglutaminol molecules. The third group of newly formed species are $(\mathrm{Pgn})_{2} \mathrm{C}_{n}^{+}, n=[42,48]$ and $(\mathrm{Pgn})_{2} \mathrm{DDC}^{+}$, formed through the collision reaction of $(\mathrm{Pgn}) \mathrm{C}_{n}^{+}$and $(\mathrm{Pgn}) \mathrm{DDC}^{+}$with pyroglutaminol molecules. Similarly, the fourth, fifth, sixth, and seventh groups of formed species are $(\mathrm{Pgn})_{3} \mathrm{C}_{n}^{+}$and $(\mathrm{Pgn})_{3} \mathrm{DDC}^{+},(\mathrm{Pgn})_{4} \mathrm{C}_{n}^{+}$ and $(\mathrm{Pgn})_{4} \mathrm{DDC}^{+},(\mathrm{Pgn})_{5} \mathrm{C}_{n}^{+}$and $(\mathrm{Pgn})_{5} \mathrm{DDC}^{+}$, and $(\mathrm{Pgn})_{6} \mathrm{C}_{n}^{+}$ and $(\mathrm{Pgn})_{6} \mathrm{DDC}^{+}$, respectively, also formed through collision reactions with pyroglutaminol molecules.

From the mass spectrum presented in Fig. 4, the intensity ratio of $(\mathrm{Pgn}) \mathrm{DDC}^{+} / \mathrm{DDC}^{+}$is smaller than $(\mathrm{Pgn}) \mathrm{C}_{n}^{+} / \mathrm{C}_{n}^{+}$, which may suggest the dehydrogenated $\mathrm{PAH}$ cations have a lower chemical reactivity than carbon cluster cations during reactions with pyroglutaminol molecules. Interestingly, $(\mathrm{Pgn})_{(1-5)} \mathrm{DDC}^{+}$ presented a higher chemical reactivity in the processing of additional adduct with pyroglutaminol molecules.

We present the photo-fragmentation and formation pathway for the DC-pyroglutaminol system: in the time range of 5.8$6.8 \mathrm{~s}$, the photo-dissociation channel upon laser irradiation for DC cations should be the same as shown above; in the time range of 6.8-9.88 s, the new cluster cations are formed through a collision formation reaction between fragmented DC cations (as shown above) and pyroglutaminol molecules. The formation reaction pathways are summarized as follows:

$$
\begin{gathered}
(\mathrm{Pgn})_{m-1} \mathrm{DDC}^{+} \stackrel{\text { Pgn }}{\longrightarrow}(\mathrm{Pgn})_{m} \mathrm{DDC}^{+}, m=[1,6] \\
(\mathrm{Pgn})_{m-1} \mathrm{C}_{n}^{+} \stackrel{\text { Pgn }}{\longrightarrow}(\mathrm{Pgn})_{m} \mathrm{C}_{n}^{+}, m=[1,6] .
\end{gathered}
$$

In all, the gas-phase reactions between large PAH species (e.g., dehydrogenated PAHs or carbon cluster molecules) and organic molecules occur relatively easily, resulting in a very large number of reaction pathways and very complex newly formed molecular clusters. Organic molecules have a different formation behavior when reacting with PAH species; from this obtained mass spectrum, we can summarize that carbon cluster cations (e.g., $\mathrm{C}_{40}-\mathrm{C}_{48}$ cations) have a higher reactivity than dehydrogenated PAH cations (e.g., DDC ${ }^{+}$). The functional group of each organic molecule may play a decisive role in their chemical reactivity, and theoretical studies regarding the formation pathway and the chemical reactivity of organic molecules with PAH species will be carried out in a subsequent paper (Yang et al., in prep.). 


\section{Discussion and astronomical implications}

The experimental results of the ion-molecule collision reactions described here suggest that large new particles can be built up under the promotion of a strong irradiation field and yield an interesting array of PAH-organic molecule clusters through aromatic-based compounds (e.g., PAHs or carbon clusters) reacting with organic molecules. The newly formed cluster contains one or several amino groups and acidic carboxyl groups; in other words, these clusters may offer a good approach for studying complex interstellar prebiotic compounds (Herbst \& van Dishoeck 2009; Jäger et al. 2009).

In the ion-molecule reaction formation processes and the reaction evolution network, the relative reactivity of these species is very important: it affects the abundance ratio of formed species (Jäger et al. 2009; Montillaud et al. 2013; Zhen et al. 2019). In this work, the relative reactivity of these species is initially obtained: dehydrogenated PAH cations have a lower reactivity than carbon cluster cations, and organic molecules have a different formation behavior when reacting with PAH species. Organic molecules contain highly reactive reaction centers because of the functional groups, which facilitates the molecular adduct aggregation. More, large isomers (e.g., $(\mathrm{Pgn})_{6} \mathrm{C}_{48}^{+}$and $\left.(\mathrm{Pgn})_{6} \mathrm{DDC}^{+}\right)$are therefore formed, ranging from 61 to 164 atoms, and the formation mechanism (gas-phase condensation) provides a possible explanation for the formation of large complex prebiotic compounds in space (Herbst \& van Dishoeck 2009; Tielens 2013).

Our obtained experimental results open up the aromaticbased chemistry that is available to the species (dehydrogenated PAHs and carbon clusters molecules) that formed from the photo-fragmentation process of PAHs in interstellar environments (Tielens 2013; Zhen et al. 2014; Castellanos et al. 2018). In the ISM, the dehydrogenated PAH cations and carbon cluster cations occur where UV photons dominate (Montillaud et al. 2013; Croiset et al. 2016). Organic molecules would have low abundances in UV-rich environments (Sorrell 2001; Herbst \& van Dishoeck 2009; Woods et al. 2012). The molecule abundance spatial distributions of PAH species and the organic molecules do not overlap, which provides constraints on the cluster formation in the ISM. Nevertheless, species formed by the reaction between PAH cations, carbon clusters cations, and organic molecules may still be involved in the ISM (Herbst \& van Dishoeck 2009). Further observational and experimental studies are required to address this issue.

Prebiotic compounds may be brought to the Earth by meteorites (e.g., Pizzarello \& Huang 2005; Ehrenfreund \& Charnley 2000). Carbonaceous meteorites contain numerous soluble organic compounds, and many of these organic compounds contain familiar biochemical species (such as amino acids, fatty acids, purines, pyrimidines, and sugars; Cronin \& Chang 1993; Botta \& Bada 2002; Snyder 2006; Furukawa et al. 2019). All the complex species discussed above are produced through ion-molecule collision reactions. Thus, it seems reasonable to hypothesize that in the ISM many of these compounds may firstly be incorporated as small dust or incorporated into small dust through gas-phase condensation by ion-molecule collision reactions, and that the small dust then grows into large dust, eventually forming comets and meteorites (Dunk et al. 2013; Delaunay et al. 2015; Zhen 2019; Gavilan et al. 2020).

\section{Conclusions}

In conclusion, we have experimentally investigated three gasphase reaction systems (DC-pyroglutamic acid, DC-proline, and
DC-pyroglutaminol) to determine the formation of astronomically relevant large PAH-organic molecule cluster cations. They reveal a general cluster formation process, that is to say, they formed a series of PAH-organic molecule cluster cations and carbon cluster-organic molecule cluster cations. Complex organic molecules have a different formation behavior when reacting with PAH species (e.g., dehydrogenated PAHs or carbon cluster molecules), and the functional group of each organic molecule may play a decisive role in their chemical reactivity. These laboratory studies attest to the importance of ion-molecule reaction synthesis routes for the chemical complexity in space and have demonstrated that the gas-phase interstellar materials could directly lead to the formation of prebiotic compounds in a bottom-up growth process. When conditions are suitable, for example when there are similar spatial distributions of molecular abundances in the interstellar cloud, prebiotic compounds can form efficiently and may be widespread in space.

Acknowledgements. This work is supported by the Strategic Priority Research Program of Chinese Academy of Sciences, Grant No. XDB 41000000 and from the National Natural Science Foundation of China (NSFC, Grant No. 12073027, and Grant No.41625013). YC acknowledges the grant from the National Natural Science Foundation of China (NSFC, Grant No. 21827804).

\section{References}

Allamandola, L. J., Tielens, A. G. G. M., \& Barker, J. R. 1989, ApJS, 71, 733 Allamandola, L. J., Joblin, C., \& Tielens, A. G. G. M. 2011, EAS Pub. Ser., 46, 305

Beltrán, M. T., Codella, C., Viti, S., et al. 2009, ApJ, 690, L93

Berné, O., Joblin, C., Deville, Y., et al. 2007, A\&A, 469, 575

Botta, O., \& Bada, J. L. 2002, Surv. Geophys. 23, 411

Castellanos, P., Candian, A., Zhen, J., et al. 2018, A\&A, 616, A166

Croiset, B. A., Candian, A., Berné, O., et al. 2016, A\&A, 590, A26

Cronin, J. R., \& Chang, S. 1993, The Chemistry of Lifes Origin (The Netherlands: Springer )

Delaunay, R., Gatchell, M., Rousseau, P., et al. 2015, J. Phys. Chem. Lett., 6, 1536

Dulieu, F., Nguyen, T., Congiu, E., et al. 2019, MNRAS, 484, L119

Dunk, P. W., Adjizian, J. J., Kaiser, N. K., et al. 2013, Proc. Nat. Acad. Sci., 110, 18081

Ehrenfreund, P., \& Charnley, S. B. 2000, ARA\&A, 38, 42

Favre, C., Fedele, D., Semenov, D., et al. 2018, ApJ, 862, L2

Furukawa, Y., Chikaraishi, Y., Ohkouchi, N., et al. 2019, Proc. Nat. Acad. Sci., 116,24440

Gavilan, L., Bejaoui, S., Haggmark, M., et al. 2020, ApJ, 889, 101

Herbst, E., \& van Dishoeck, E. F. 2009, ARA\&A, 47, 427

Hollis, J. M., Lovas, F. J., Jewell, P. R. 2000, ApJ, 540, L107

Jäger, C., Huisken, F., Mutschke, H., et al. 2009, ApJ, 696, 706

Lifshitz, C. 2000, IJMS, 200, 423

McGuire, B. A., Loomis, R. A., Burkhardt, A. M., et al. 2021, Science, 371, 1265

Montillaud, J., Joblin, C., \& Toublanc, D. 2013, A\&A, 552, A15

Pilleri, P., Joblin, C., Boulanger, F., et al. 2015, A\&A, 577, A16

Pizzarello, S., \& Huang, Y. 2005, Geochim. Cosmochim. Acta, 69, 599

Pizzarello, S., Cooper, G. W., \& Flynn, G. J. 2006, Meteorites and the Early Solar System II (Tucson: University of Arizona Press)

Puget, J. L., \& Leger, A. 1989, ARA\&A, 27, 161

Rapacioli, M., Joblin, C., \& Boissel, P. 2005, A\&A, 429, 193

Rapacioli, M., Calvo, F., Joblin, C., et al. 2006, A\&A, 460, 519

Rhee, Y. M., Lee, T. J., Gudipati, M. S., et al, 2007, Proc. Nat. Acad. Sci., 104, 5274

Sellgren, K. 1984, ApJ, 277, 623

Snyder, L. E. 1997, Origins Life Evol. Biosphere, 27, 115

Snyder, L. E. 2006, Proc. Natl. Acad. Sci., 103, 12243

Sorrell, W. H. 2001, ApJ, 555, L129

Tielens, A. G. G. M. 2013, Rev. Mod. Phys., 85, 1021

van Diedenhoven, B., Peeters, E., Van Kerckhoven, C., et al. 2004, ApJ, 611, 928

Woods, P. M., Kelly, G., Viti, S., et al. 2012, ApJ, 750, 19

Zhen, J. 2019, A\&A, 623, A102

Zhen, J., Castellanos, P., Paardekooper, D. M., et al. 2014, ApJ, 797, L30

Zhen, J., Candian, A., Castellanos, P., et al. 2018, ApJ, 854, 27

Zhen, J., Zhang, W., Yang, Y., et al. 2019, ApJ, 887, 70 